\title{
SEMI-IMPLICIT RATIONAL RUNGE- KUTTA METHOD OF SOLVING SECOND ORDER DIFFERENTIAL EQUATION
}

\author{
Eric Augustine Oje \\ Department of Mathematical Sciences \\ Taraba State University, Jalingo, Taraba State, \\ Nigeria
}

\begin{abstract}
There is much work done on implicit and explicit rational Runge-Kutta schemes for second order ordinary differential equations. This motivated the need for semi-implicit scheme since no work has been done on it for second order ordinary differential equations. The research was carried out using Taylor"es series and Binomial expansion after some related works were reviewed in the literature. The research was completed in which a one-stage semi-implicit rational RungeKutta scheme was derived, the convergence and consistency of the scheme was tested and the scheme was convergent and consistent. The adopted scheme was discovered to perform well in terms of approximating the exact solution of a special case of second order ordinary differential equations.
\end{abstract}

Keywords - ordinary differential equations, Runge-Kutta, semi-implicit scheme

\section{INTRODUCTION}

An ordinary differential equation (ODE) is an equation of the form:

$$
y^{\prime}=f(x, y) \quad<b^{y\left(x_{0}\right)=y_{0} \quad a<x}
$$

Arising from mathematical modeling of a large variety of physical problems in nuclear reactions, delay problems and computer aided designs, perturbation problems or dynamical processes in industries, technological fields and economy of some third world countries affected by inflation and other economic depressions. Often, the solution $y(x)$ of the ordinary differential equation (1) possesses components with fast and slow responses and one in which there is pole or discontinuities. The existing methods of solving this ordinary differential equation includes: Runge-Kutta method, Euler's method, implicit backward difference method proposed by Gear in 1971, Hybrid methods by Ademiluyi (1987),

\author{
Aggrey Eric Majuk \\ Department of Computer Graphics and Image \\ Processing \\ University of Debrecen, Debrecen, Hungary
}

perturbed polynomial process by Lambert and Shaw, extrapolation process by Evans and Fatunla, Rational functions approximation method by Luke et al.

Runge-Kutta schemes are important family of implicit and explicit iterative methods for approximation of solution of ordinary differential equations. Consider the numerical approximation of second order initial value problems of the form:

$$
\begin{aligned}
& y^{\prime \prime}=f\left(x, y, y^{\prime}\right), \quad y\left(x_{0}\right)=y_{0}, \quad y^{\prime}\left(x_{0}\right) \\
& =y_{0}, \quad a \leq x \leq b
\end{aligned}
$$

The general s- stage Runge-Kutta scheme for general second order initial value problems of ordinary differential equations of the form (1) as defined by Jain (1984) is:

$$
\begin{aligned}
& y_{n+1} \\
& =y_{n}+h y^{\prime} \\
& +\sum_{r=1}^{s} c_{r} k_{r}
\end{aligned}
$$

And

$$
\begin{aligned}
& y^{\prime}{ }_{n+1} \\
& =y^{\prime}{ }_{n_{s}} \\
& +\frac{1}{h} \sum_{r=1}^{{ }_{n}} c^{\prime}{ }_{r} k_{r}
\end{aligned}
$$

Where 
small values of s (Sharp et al, 1992 and Dormand et

$$
\begin{gathered}
k_{r}=\frac{h^{2}}{2} f\left(x_{n}+c_{i} h, y_{n}+h c_{i} y_{n}^{\prime}+\sum_{j=1}^{r} a_{i j} k_{j}, y^{\prime}{ }_{n}\right. \\
\left.+\frac{1}{h} \sum_{j=1}^{\infty} b_{i j} k_{j}\right), \quad i(1) s
\end{gathered}
$$

With

$\frac{1}{2} \sum_{j=1}^{i} b_{i j}, \quad i(1) r$

$$
c_{i}=\sum_{j=1}^{i} a_{i j}=
$$

Where $c_{i}, a_{i j}, b_{i j}, c_{r}, c^{\prime}{ }_{r}$ are constants to be determined. The suitable parameter requires extremely lengthy algebraic manipulation, except for al, 1987).

In 1982, Hong Yuan Fu proposed a Rational Runge Kutta scheme for the integration of this kind of ordinary differential equations. Okunbor (1985), Babatola and Ademiluyi (2000) and Bolaji (2005) worked further on this method and proposed schemes that can be classified as explicit, implicit or semi implicit method. Bolarinwa et al (2012) proposed a two-stage semi-implicit rational RungeKutta scheme for solving ordinary differential (5quation of first order.

From the above, the researcher will focus on developing a scheme for semi-implicit rational Runge-Kutta method of solving ordinary differential equation of second order.

\section{DeRIVATION OF THE Scheme}

The rational form of (2.1) and (2.2) can be defined as

$$
\begin{aligned}
& y_{n+1}=\frac{y_{n}+h y_{n}^{\prime}+\sum_{i=1}^{r} w_{i} k_{i}}{1+y_{n} \sum_{i=1}^{r} v_{i} H_{i}} \\
& y_{n+1}^{\prime}=\frac{y_{n}^{\prime}+\frac{1}{h} \sum_{i=1}^{r} w_{r}^{\prime} k_{i}}{1+\frac{1}{h} y_{n}^{\prime} \sum_{i=1}^{r} v_{i}^{\prime} H_{i}} \\
& k_{i}=\frac{h^{2}}{2} f\left(x_{n}+c_{i} h, y_{n}+h c_{i} y_{n}^{\prime}+\sum_{j=1}^{i} a_{i j} k_{j}, y_{n}^{\prime}+\frac{1}{h} \sum_{j=1}^{i} b_{i j} k_{j}\right), i=1(1) r \\
& H_{i}=\frac{h^{2}}{2} g\left(x_{n}+d_{i} h, z_{n}+h d_{i} z_{n}^{\prime}+\sum_{j=1}^{i} \alpha_{i j} H_{j}, z_{n}^{\prime}+\frac{1}{h} \sum_{j=1}^{i} \beta_{i j} H_{j}\right), i=1(1) r
\end{aligned}
$$

with constraints

$$
c_{i}=\sum_{j=1}^{i} a_{i j}=\frac{1}{2} \sum_{j=1}^{i} b_{i j}, i=1(1) r
$$

$$
d_{i}=\sum_{j=1}^{i} \alpha_{i j}=\frac{1}{2} \sum_{j=1}^{i} \beta_{i j}, i=1(1) r
$$

in which 


\section{International Journal of Engineering Applied Sciences and Technology, 2020 \\ Vol. 4, Issue 9, ISSN No. 2455-2143, Pages 104-115 \\ Published Online January 2020 in IJEAST (http://www.ijeast.com)}

$$
g\left(x_{n}, z_{n}\right)=-z_{n}^{2} f\left(x_{n}, y_{n}\right) \operatorname{and} z_{n}=\frac{1}{y_{n}}
$$

The constraint equations are to ensure consistency of the method, $\mathrm{h}$ is the step size and the parameters $a_{i j}, b_{i j}, c_{i}, d_{i}, \propto_{i j}, \beta_{i j}$ are constants called the parameters of method.

Following Abhulimen and Uloku(2012), Bolarinwa(2012) procedures:

i. Obtain the taylor series expansion of $k_{i}$ and $H_{i}$ about the point $\left(x_{n}, y_{n}, y_{n}^{\prime}\right)$ and binomial series expansion of righthand side of (2.7) and (2.8).

ii. Insert the Taylor series expansion into (2.7) and (2.8) respectively.

iii. Compare the final expansion of $k_{i}$ and $H_{i}$ about the point $\left(x_{n}, y_{n}, y_{n}^{\prime}\right)$ to the Taylor series expansion of $y_{n+1}$ and $y_{n+1}^{\prime}$ about $\left(x_{n}, y_{n}, y_{n}^{\prime}\right)$ in the powers of $h$.

Normally the number of parameters exceed the number of equations, these parameters are chosen to ensure that (one or more of) the following conditions are satisfied.

i. Minimum bound of local truncation error exists

ii. The method has maximized interval of absolute stability

iii. Minimized computer storage facilities are utilized.

In equations (2.5),(2.6),(2.7) and (2.8), setting $\mathrm{r}=1$ we have:

$$
\begin{gathered}
y_{n+1}=\frac{y_{n}+h y^{\prime}+w_{1} k_{1}}{1+y_{n} v_{1} H_{1}} \\
y_{n+1}^{\prime}=\frac{y_{n}^{\prime}+\frac{1}{h} w_{1}^{\prime} k_{1}}{1+\frac{1}{h} y^{\prime}{ }_{n} v_{1}^{\prime} H_{1}}
\end{gathered}
$$

where

$$
\begin{gathered}
k_{i}=\frac{h^{2}}{2} f\left(x_{n}+c_{1} h, y_{n}+h c_{1} y_{n}^{\prime}+\sum_{j=1}^{1} a_{i j} k_{j}, y^{\prime}{ }_{n}+\frac{1}{h} \sum_{j=1}^{1} b_{i j} k_{j}\right), i=1 \\
H_{i}=\frac{h^{2}}{2} g\left(x_{n}+d_{1} h, z_{n}+h d_{1} z_{n}^{\prime}+\sum_{j=1}^{1} \propto_{i j} H_{j}, z^{\prime}{ }_{n}+\frac{1}{h} \sum_{j=1}^{1} \beta_{i j} H_{j}\right), i=1
\end{gathered}
$$

with constraints

$$
\begin{array}{r}
c_{1}=a_{11}=\frac{1}{2} b_{11} \\
\\
d_{1}=\propto_{11}=\frac{1}{2} \beta_{11}
\end{array}
$$




\section{International Journal of Engineering Applied Sciences and Technology, 2020 \\ Vol. 4, Issue 9, ISSN No. 2455-2143, Pages 104-115 \\ Published Online January 2020 in IJEAST (http://www.ijeast.com)}

since we are considering the semi-implicit scheme, $b_{i j}=0$ for $j>i$.

Where $a_{i j}, b_{i j}, c_{i}, d_{i}, \propto_{i j}, \beta_{i j}, w_{1}, w_{1}^{\prime}, v_{1}, v_{1}^{\prime}$ are all constants to be determined.

Now by adopting a binomial expansion (definition 1.6.10) on equations (2.9) gives

$$
\begin{aligned}
y_{n+1} & =\left(y_{n}+h y_{n}^{\prime}+w_{1} k_{1}\right)\left(1+y_{n} v_{1} H_{1}\right)^{-1} \\
& =\left(y_{n}+h y_{n}^{\prime}+w_{1} k_{1}\right)\left(1-y_{n} v_{1} H_{1}\right) \\
& =y_{n}+h y_{n}^{\prime}-\left(y_{n}^{2} v_{1}+h y_{n} y_{n}^{\prime} v_{1}\right) H_{1}+\left(w_{1}-y_{n} v_{1} H_{1} w_{1}\right) k_{1}
\end{aligned}
$$

Similarly the binomial expansion of (2.10) gives

$$
\begin{aligned}
y_{n+1}^{\prime} & =\left(y_{n}^{\prime}+\frac{1}{h} w_{1}^{\prime} k_{1}\right)\left(1+\frac{1}{h} y_{n}^{\prime} v_{1}^{\prime} H_{1}\right)^{-1} \\
& =\left(y^{\prime}{ }_{n}+\frac{1}{h} w_{1}^{\prime} k_{1}\right)\left(1-\frac{1}{h} y^{\prime}{ }_{n} v_{1}^{\prime} H_{1}\right) \\
& =y_{n}^{\prime}+\frac{1}{h} w_{1}^{\prime} k_{1}-\left(\frac{1}{h} y_{n}^{\prime 2} v_{1}^{\prime}+\frac{1}{h^{2}} y_{n}^{\prime} w_{1}^{\prime} v_{1}^{\prime} k_{1}\right) H_{1}
\end{aligned}
$$

Now, the Taylor's series expansion of $y_{n+1}$ about $x_{n}$ gives

$$
y_{n+1}=y_{n}+h y_{n}^{\prime}+\frac{h^{2}}{2 !} y_{n}^{\prime \prime}+\frac{h^{3}}{3 !} y_{n}^{\prime \prime \prime}+\frac{h^{4}}{4 !} y_{n}^{i v}+\cdots
$$

and

$y_{n+1}^{\prime}=y_{n}^{\prime}+h y_{n}^{\prime \prime}+\frac{h^{2}}{2 !} y_{n}^{\prime \prime \prime}+\frac{h^{3}}{3 !} y_{n}^{i v}+\cdots$

where

$$
\begin{aligned}
& y_{n}^{\prime \prime}=f\left(x_{n}, y_{n}, y_{n}^{\prime}\right)=f_{n} \\
& y_{n}^{\prime \prime \prime}=f_{x}+y^{\prime} f_{y}+f_{n} f_{y^{\prime}}=\Delta f_{n} \\
& y_{n}^{i v}=f_{x x}+y_{n}^{\prime 2} f_{y y}+f^{2} f_{y^{\prime} y^{\prime}}+2 y^{\prime} f_{n} f_{y y^{\prime}}+2 f_{n} f_{x y^{\prime}}+f_{y^{\prime}} \Delta f_{n}=\Delta^{2} f_{n}+f_{y^{\prime}} \Delta f_{n}+f_{n} f_{y} \\
& \text { since } \Delta=\frac{\partial}{\partial x}+y^{\prime} \frac{\partial}{\partial y}+f_{n} \frac{\partial}{\partial y^{\prime}}
\end{aligned}
$$

Using the Taylor's series of the function of three variables (definition 1.6.8) we have from (2.11), 


$$
\begin{aligned}
\frac{2}{h^{2}} k_{1}=f_{n}+\left(c_{1} h f_{x}+\left(h c_{1} y_{n}^{\prime}+a_{11} k_{1}\right) f_{n}+\frac{1}{h} b_{11} k_{1} f_{y^{\prime}}\right) & \\
+ & \frac{1}{2 !}\left[\left(c_{1} h\right)^{2} f_{x x}+2 c_{1} h\left(h c_{1} y_{n}^{\prime}+a_{11} k_{1}\right) f_{x y}+2 c_{1} h\left(\frac{1}{h} b_{11} k_{1}\right) f_{x y^{\prime}}+\left(h c_{1} y_{n}^{\prime}+a_{11} k_{1}\right)^{2} f_{y y}\right. \\
+ & \left.2\left(h c_{1} y_{n}^{\prime}+a_{11} k_{1}\right)\left(\frac{1}{h} b_{11} k_{1}\right) f_{y y^{\prime}}+\left(\frac{1}{h} b_{11} k_{1}\right)^{2} f_{y^{\prime} y^{\prime}}\right]+\cdots
\end{aligned}
$$

simplifying further and re-arranging the equation in powers of $\mathrm{h}$ gives

$$
\begin{aligned}
k_{1}=\frac{h}{2}\left[b_{11} k_{1} f_{y y^{\prime}}\right. & \left.+a_{11} b_{11} k_{1}^{2} f_{y y^{\prime}}\right]+\frac{h^{2}}{2}\left[f_{n}+a_{11} k_{1} f_{y}+c_{1} b_{11} k_{1} f_{x y^{\prime}}+\frac{1}{2} a_{11}^{2} k_{1}^{2} f_{y y}+c_{1} y_{n}^{\prime} b_{11} k_{1} f_{y y^{\prime}}\right] \\
& +\frac{h^{3}}{2}\left[c_{1} f_{x}+c_{1} y_{n}^{\prime} f_{y}+c_{1} y_{n}^{\prime} f_{y}+c_{1} a_{11} k_{1} f_{x y}+c_{1} a_{11} y_{n}^{\prime} k_{1} f_{y y}\right] \\
& +\frac{h^{4}}{4}\left[c_{1}^{2} f_{x x}+2 c_{1}^{2} y_{n}^{\prime} f_{x y}+c_{1}^{2} y_{n}^{\prime 2} f_{y y}\right]+0\left(h^{3}\right)(2.15)
\end{aligned}
$$

Equation (2.15) is implicit, which cannot be proceeded by successive substitutions, we assume a solution of $\mathrm{k}_{1}$ which may be expressed as

$$
k_{1}=h A_{1}+h^{2} B_{1}+h^{3} C_{1}+h^{4} D_{1}+O\left(h^{3}\right)
$$

be able to compare and evaluate coefficients of $h$

Substituting the values of $\mathrm{k}_{1}$ of (2.16) into (2.15) expand and re-arranging in powers of $h$ gives

$$
\begin{aligned}
k_{1}=\frac{h}{2}\left[b _ { 1 1 } \left(h A_{1}+\right.\right. & \left.\left.h^{2} B_{1}+h^{3} C_{1}\right) f_{y^{\prime}}+a_{11} b_{11}\left(h A_{1}+h^{2} B_{1}\right)^{2} f_{y y^{\prime}}\right] \\
& +\frac{h^{2}}{2}\left[f_{n}+a_{11}\left(h A_{1}+h^{2} B_{1}\right) f_{y}+C_{1} b_{11}\left(h A_{1}+h^{2} B_{1}\right) f_{x y^{\prime}}+a_{11}^{2}\left(h A_{1}\right)^{2} f_{y y}\right. \\
& \left.+C_{1} y_{n}^{\prime} b_{11}\left(h A_{1}+h^{2} B_{1}\right) f_{y y^{\prime}}\right]+\frac{h^{3}}{2}\left[c_{1} f_{x}+c_{1} y_{n}^{\prime} f_{y}+c_{1} a_{11}\left(h A_{1}\right) f_{x y}+c_{1} a_{11} y_{n}^{\prime}\left(h A_{1}\right) f_{y y}\right] \\
& +\frac{h^{4}}{4}\left[c_{1}^{2} f_{x x}+c_{1}^{2} y_{n}^{\prime} f_{x y}+c_{1}^{2} y_{n}^{\prime 2} f_{y y}\right]+0\left(h^{5}\right)(2.17)
\end{aligned}
$$

On equating the powers of $h$ from (2.16) and (2.17) gives 
$A_{1}=0$

$$
\begin{gathered}
B_{1}=\frac{1}{2} f_{n} \\
C_{1}=\frac{1}{2}\left[c_{1} f_{x}+c_{1} y_{n}^{\prime} f_{y}+\frac{1}{2} b_{11} f_{n} f_{y^{\prime}}\right]=\frac{1}{2} C_{1} \Delta f_{n} \\
D_{1}=\frac{1}{4}\left[c_{1}^{2} \Delta^{2} f_{n}+b_{11} \Delta f_{n} f_{y^{\prime}}+a_{11} f_{n} f_{y}\right]
\end{gathered}
$$

Then

$$
k_{1}=\frac{h^{2}}{2} f_{n}+\frac{h^{3}}{2} C_{1} \Delta f_{n}+\frac{h^{4}}{4}\left[c_{1}^{2} \Delta^{2} f_{n}+b_{11} \Delta f_{n} f_{y^{\prime}}+a_{11} f_{n} f_{y}\right]
$$

In a similar manner

$H_{1}=h^{2} M_{1}+h^{3} N_{1}+h^{4} R_{1}+0\left(h^{3}\right)(2.20)$

where

$$
\begin{array}{r}
M_{1}=\frac{1}{2} g_{n} \\
N_{1}=\frac{1}{2} d_{1} \Delta g_{n} \\
R_{1}=\frac{1}{4}\left[d_{1}^{2} \Delta^{2} g_{n}+\beta_{11} \Delta g_{n} g_{z^{\prime}}+\alpha_{11} g_{n} g_{z}\right]
\end{array}
$$

and also

$$
H_{1}=\frac{h^{2}}{2} g_{n}+\frac{h^{3}}{2} d_{1} \Delta g_{n}+\frac{h^{4}}{4}\left[d_{1}^{2} \Delta^{2} g_{n}+\beta_{11} \Delta g_{n} g_{z^{\prime}}+\alpha_{11} g_{n} g_{z}\right]
$$

Substituting 2.16 and 2.20 into 2.13 and 2.14

$$
\begin{gathered}
y_{n+1}=y_{n}+h y_{n}^{\prime}-\left(y_{n}^{2} v_{1}+h y_{n} y_{n}^{\prime} v_{1}\right)\left(h^{2} M_{1}+h^{3} N_{1}+h^{4} R_{1}\right)+\left(w_{1}-y_{n} v_{1} H_{1} w_{1}\right)\left(h^{2} B_{1}+h^{3} C_{1}\right. \\
\left.+h^{4} D_{1}\right)
\end{gathered}
$$

Expanding the brackets and re-arranging in powers of $h$ gives

$$
y_{n+1}=y_{n}+h y_{n}^{\prime}+h^{2}\left(w_{1} B_{1}-y_{n}^{2} v_{1} M_{1}\right)+h^{3}\left(w_{1} C_{1}-y^{2} v_{1} N_{1}-y_{n} y_{n}^{\prime} v_{1} M_{1}\right)+0\left(h^{3}\right)
$$

also for 


$$
\begin{gathered}
\begin{array}{c}
y_{n+1}^{\prime}=y_{n}^{\prime}+\frac{1}{h} w_{1}^{\prime}\left(h^{2} B_{1}+h^{3} C_{1}+h^{4} D_{1}\right)-\left(\frac{1}{h} y_{n}^{\prime 2} v_{1}^{\prime}+\frac{1}{h^{2}} y_{n}^{\prime} w_{1}^{\prime} v_{1}^{\prime}\left(h^{2} B_{1}+h^{3} C_{1}+h^{4} D_{1}\right)\right)\left(h^{2} M_{1}+h^{3} N_{1}\right. \\
\left.+h^{4} R_{1}\right) \\
\text { Expanding the brackets and re-arranging in powers of h gives } \\
y_{n+1}^{\prime}=y_{n}^{\prime}+h\left(w_{1}^{\prime} B_{1}-y_{n}^{2} v_{1}^{\prime} M_{1}\right)+h^{2}\left(w_{1}^{\prime} C_{1}-y_{n}^{\prime 2} v_{1}^{\prime} N_{1}-y_{n}^{\prime} w_{1}^{\prime} v_{1}^{\prime} B_{1} M_{1}\right) \\
+h^{3}\left(w_{1}^{\prime} D_{1}-y_{n}^{\prime 2} v_{1}^{\prime} R_{1}-y_{n}^{\prime} w_{1}^{\prime} v_{1}^{\prime} B_{1} N_{1}-y_{n}^{\prime} w_{1}^{\prime} v_{1}^{\prime} C_{1} M_{1}\right)+0\left(h^{3}\right) \\
\text { Comparing the corresponding powers in } h
\end{array} \\
\begin{array}{c}
\frac{1}{2} w_{1} f_{n}-\frac{1}{2} y_{n}^{2} v_{1} g_{n}=\frac{1}{2} f_{n} \quad \\
\frac{1}{2} w_{1} C_{1} \Delta f_{n}-\frac{1}{2} y_{n}^{\prime 2} v_{1} d_{1} \Delta g_{n}-\frac{1}{2} y_{n} y_{n}^{\prime} v_{1} g_{n}=\frac{1}{6} \Delta f_{n} \\
\frac{1}{2} w_{1}^{\prime} f_{n}-\frac{1}{2} y_{n}^{2} y_{n}^{\prime} g_{n}=f_{n} \\
\frac{1}{2} w_{1}^{\prime} \Delta f_{n}-\frac{1}{2} y_{n}^{\prime 2} v_{1}^{\prime} d_{1} \Delta g_{n}-\frac{1}{2} y_{n}^{\prime} w_{1}^{\prime} v_{1}^{\prime} f_{n}\left(\frac{1}{2} g_{n}\right)=\frac{1}{2} \Delta f_{n}
\end{array}
\end{gathered}
$$

Expanding the brackets and re-arranging in powers of $\mathrm{h}$ gives

$$
\begin{aligned}
y_{n+1}^{\prime}=y_{n}^{\prime}+h\left(w_{1}^{\prime} B_{1}-y_{n}^{2} v_{1}^{\prime} M_{1}\right)+h^{2}\left(w_{1}^{\prime} C_{1}-y_{n}^{\prime 2} v_{1}^{\prime} N_{1}-y_{n}^{\prime} w_{1}^{\prime} v_{1}^{\prime} B_{1} M_{1}\right) \\
+h^{3}\left(w_{1}^{\prime} D_{1}-y_{n}^{\prime 2} v_{1}^{\prime} R_{1}-y_{n}^{\prime} w_{1}^{\prime} v_{1}^{\prime} B_{1} N_{1}-y_{n}^{\prime} w_{1}^{\prime} v_{1}^{\prime} C_{1} M_{1}\right)+0\left(h^{3}\right)
\end{aligned}
$$

Comparing the corresponding powers in $h$

By using equation

$$
g_{n}=-\frac{f_{n}}{y_{n}^{2}}, g_{x}=-\frac{f_{x}}{y_{n}^{2}} g_{z}=-2 \frac{f_{n}}{y_{n}}+f_{y}, \quad g_{z^{\prime}}=-2 \frac{f_{n}}{y_{n}}+f_{y^{\prime}}, \quad z_{n}^{\prime}=-\frac{y_{n}^{\prime}}{y_{n}^{2}}
$$

$\Delta g_{n}=g_{n}+z_{n}^{\prime} g_{n}+g_{n} g_{z^{\prime}}$

using 2.24 into 2.23 we get the following simultaneous equations

$w_{1}+v_{1}=1$

$w_{1} c_{1}+v_{1} d_{1}=\frac{1}{3}$

$w_{1}^{\prime}+v_{1}^{\prime}=2$

$w_{1}^{\prime} c_{1}+v_{1}^{\prime} d_{1}=1$

Equation (2.25) has four (4) with six (6) unknowns; there will not be a unique solution, we choose $d_{1}$ to be zero in order to obtain a semi-implicit scheme. There will be a family of one-stage scheme.

Choosing the parameters

By solving (2.25) the following values were obtained so the equation 2.25 is satisfied and makes the scheme semi-implicit

$$
\begin{aligned}
& w_{1}=\frac{2}{3}, v_{1}=\frac{1}{3}, c_{1}=\frac{1}{2}, a_{11}=\frac{1}{2}, b_{11}=1, d_{1}=\alpha_{11}=\beta_{11}=0, \\
& w_{1}^{\prime}=2, v_{1}^{\prime}=0
\end{aligned}
$$


the scheme obtained is $y_{n+1}=\frac{y_{n}+h y_{n}^{\prime}+\frac{2}{3} k_{1}}{1+\frac{1}{3} y_{n} H_{1}}$

and

$y_{n+1}^{\prime}=y_{n}^{\prime}+\frac{2}{h} k_{1}$

where

$k_{1}=\frac{h^{2}}{2} f\left(x_{n}+\frac{1}{2} h, y_{n}+\frac{1}{2} h y_{n}^{\prime}+\frac{1}{2} k_{1}, y_{n}^{\prime}+\frac{1}{h} k_{1}\right)$

$H_{1}=\frac{h^{2}}{2} g\left(x_{n}, z_{n}, z_{n}^{\prime}\right)$

The schemes are semi-implicit as required, since $\mathrm{k}_{1}$ is implicit and $\mathrm{H}_{1}$ is explicit.

Choosing the parameters

$$
\begin{gathered}
w_{1}=\frac{1}{3}, v_{1}=\frac{2}{3}, c_{1}=a_{11}=\frac{1}{2}, b_{11}=1, \text { since } c_{1}=a_{11}=\frac{1}{2} b_{11}, w_{1}^{\prime}=2, v_{1}^{\prime}=0, d_{1}=\alpha_{11} \\
=\frac{1}{4}, \text { but } d_{1}=\alpha_{11}=\frac{1}{2} \beta_{11}=>\beta_{11}=\frac{1}{2}
\end{gathered}
$$

By solving (2.25) the following values were obtained so the equation 2.25 is satisfied and makes the scheme semi-implicit

The following scheme is obtained

$y_{n+1}=\frac{y_{n}+h y_{n}^{\prime}+\frac{1}{3} k_{1}}{1+\frac{2}{3} y_{n} H_{1}}$

and

$y_{n+1}^{\prime}=y_{n}^{\prime}+\frac{2}{h} k_{1}$

where

$$
\begin{aligned}
& k_{1}=\frac{h^{2}}{2} f\left(x_{n}+\frac{1}{2} h, y_{n}+\frac{h}{2} y_{n}^{\prime}+\frac{1}{2} k_{1}, y_{n}^{\prime}+\frac{1}{h} k_{1}\right) \\
& H_{1}=\frac{h^{2}}{2} g\left(x_{n}+\frac{1}{4} h, z_{n}+\frac{h}{4} z_{n}^{\prime}+\frac{1}{4} H_{1}, z_{n}^{\prime}+\frac{1}{2 h} H_{1}\right)
\end{aligned}
$$




\section{International Journal of Engineering Applied Sciences and Technology, 2020}

Vol. 4, Issue 9, ISSN No. 2455-2143, Pages 104-115

Published Online January 2020 in IJEAST (http://www.ijeast.com)

\section{NUMERICAL PROBLEMS}

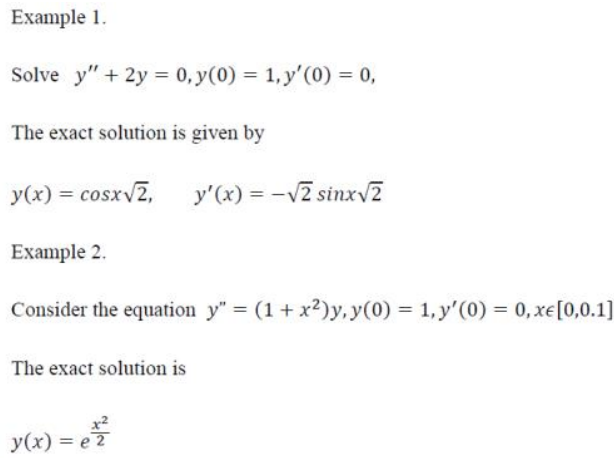

\section{Discussion OF RESUlts}

The first example 1 is an initial valued second order differential equation. This equation suits the scheme (3.26) as it is a second order ordinary differential equation of the form $\mathrm{f}(\mathrm{y})$ that is it is an autonomous system. The results get better with decrease in step size (h) as shown in table 1 in appendix A. 24

The table 2 in appendix $A$ is the result obtained by applying the schemes (3.26) to example 2 evaluated from step size $h=0.01$, as it is a special case of second order ordinary differential equation of the form $f(x, y)$. The result shows that the scheme performed well as the approximate solution approaches the exact solution better as the step size reduces to $h=0.001$ this implies the smaller the value of the step size, the better the approximated solution. This scheme approximates the special second order differential equation better as it is shown in table 2 in appendix A.

\section{CONCLUSION}

The semi-implicit rational Runge-Kutta scheme obtained in this research performed well in approximating the exact solutions and the result of the approximated solution gets better as the step size tends to zero, as it is shown in the result from table 2 in appendix A. The scheme is convergent as well as it is also consistent. This result implies that the scheme does better approximation on the special second order differential equation.

\section{REFRENCES}

1. Abhulimen, C.E. and Uloko, J.A (2012). A Class of an Implicit Stage-two Rational Runge-Kutta Method for Solution of Ordinary Differential Equations. Journal of Applied Mathematics and Bioinformatics. 2(3): 17-31
2. Ademiluyi, R.A. (1987) "A Class of Hybrid Methods for the Solution of Ordinary Differential Equations". M.sc thesis, University of Benin, Benin city; Nigeria. Unpublished.

3. Ademuluyi, R.A., Babatola, P.O. (2000). "Implicit Rational Runge-Kutta Scheme for Integration of Stiff ODEs". Nigeria Mathematical Society, (NMS) Journal.

4. Bolarinwa, B., Ademiluyi R. A., Oluwagunwa A. P. and Awomuse B. O. (2012). "A Class of Two-Stage SemiImplicit Rational Runge - Kutta Scheme for Solving Ordinary Differential Equations". Canadian Journal of Science and Engineering Mathematics, 3(3):99 - 111.

5. Bolarinwa, B. (2005). "A Class of SemiImplicit Rational Runge-Kutta Scheme for Solving Ordinary Differential Equations with Derivative Discontinuities"; M. Tech Thesis, Federal University of Technology, Akure; Nigeria. Unpublished.

6. Dass, H.K. (2012). Advance Engineering Mathematics. Third edition. S. Chand and Company Limited. New Delhi, India

7. Dormand, J.R., El-Milkawy, M.E.A, and Prince, P.J.(1987) "Families of RungeKutta-Nystrom formula", IMA Journal of Numerical Analysis: 7: 235-250

8. Dresner, L. (1999), Applications of Lie ${ }^{e c}$ s Theory of Ordinary and Partial Differential Equations, Bristol and Philadelphia: Institute of Physics Publishing.

9. Fudziah, I. (2003). Embedded Singly Diagonal Implicit Runge-Kutta-Nystrom Method Order 5(4) for the Integration of Special Second Order Ordinary 27

10. Obimba, D.A. (1993). Basic Linear Differential Equations. First Edition. Oganiru Publishing Company, Owerri, Nigeria.

11. Odekunle, M.R. (2001). "Some Semiimplicit Rational Runge-Kutta Schemes". Bagale Journal of Pure and Applied Sciences, 1(1):11-14 


\section{International Journal of Engineering Applied Sciences and Technology, 2020 \\ Vol. 4, Issue 9, ISSN No. 2455-2143, Pages 104-115 \\ Published Online January 2020 in IJEAST (http://www.ijeast.com)}

12. Okunbor, D.I. (1985); "Runge-Kutta Schemes for Stiff ODEs" M.sc Thesis, University of Benin, Nigeria.

13. Okunuga, S.A., Sofoluwa, A.B., Ehigie, J.O. and Akambi, M.A. (2012). Fifth Order TwoStage Explicit Runge-Kutta-Nystrom Method for Direct Integration 28

14. Fudziah, I. (2009). Sixth Order Singly Diagonally Implicit Runge-Kutta Nystrom Method With Explicit First Stage for Solving Second Order Ordinary Differential Equations. European Journal of Scientific Research. 26(4):470-479.

15. Gear, C.W. (1971). "DIFSUB for Solution of ODEs" Math comp. vol. 4 page 185-190. Graham, R., Knuth, D., Patashnik, O. (1994), “(5) Binomial Coefficients" Concrete Mathematics (2nd ed.) Addison Wesley.

16. Greenberg, M. (1998). Advanced Engineering Mathematics (2nd ed.), Prentice Hall.

17. Hong, Y.F. (1982): "A Class Of A-Stage Explicit Scheme, Computational and Asymptotic Method for Boundary and Interior Layer". Proceeding of ball 11 Conference, Trinity College Dublin, 236241.

18. Jain, M.K. (1984). "Numerical Solution of Differential Equations, 2nd Edition". Wiley Estem Limited. New Delhi, India.

19. Lambert, J.D. (1973). Computational Methods in Ordinary Differential Equations. New York: John Wiley and sons.

20. Oye, N.D., Adee, S.O., Odekunle, M.R., (2004). "A Class of Inverse Runge-Kutta
Schemes for the Numerical Integration of Singular Problems". Journal of Applied Mathematics and Computing. Elsevier: 158: $149-158$

21. Raisinghania, M.D. (2012). Ordinary and Partial Differential Equation. Fourth edition. New Delhi, India.

22. Senu, N., Sulaiman, M., Ismail, F. and Othman, M. (2011). A Singly Diagonally Implicit Runge-Kutta-Nystrom Method for Solving Oscillatory Problems. International Journal of Applied Mathematics. 4(1);

23. Sharp, P.W. and Fine, J.M. (1992). "Nystrom Pairs for the General Second Order Initial-Value Problems", Journal of Computational and Applied Mathematics 42: 279-291

24. Stroud, K.A. and Dexter, J.B. (2007). Advance Engineering Mathematics, Sixth Edition. Palgave Macmillan Company, New York.

25. Usman, A.S., Odekunle, M.R. and Ahmad, M.M. (2013). "A Class of Three Stage Implicit Rational Runge-Kutta Schemes for Approximation of Second Order Ordinary Differential Equations". The International Institute for Science, Technology and Education. 3(11), 121130. 


\section{International Journal of Engineering Applied Sciences and Technology, 2020 \\ Vol. 4, Issue 9, ISSN No. 2455-2143, Pages 104-115 \\ Published Online January 2020 in IJEAST (http://www.ijeast.com)}

\section{APPENDIX A}

Table 1: Solution of Example 1 at $\mathrm{h}=0.001$

\begin{tabular}{|c|c|c|c|c|c|c|}
\hline & Exact & solutions & Approximated & solutions & Error & \\
\hline $\mathrm{H}$ & $\mathrm{y}(\mathrm{x})$ & $\mathrm{y}^{\prime}(\mathrm{x})$ & $\mathrm{y}(\mathrm{x})$ & $\mathrm{y}^{\prime}(\mathrm{x})$ & $\mathrm{y}$ & $\mathrm{y}^{\prime}$ \\
\hline 0.001 & 0.99001665 & -0.19933400 & 1.00000000 & -0.00199999 & $9.98 \mathrm{E}-03$ & $1.97 \mathrm{E}-01$ \\
\hline 0.002 & 0.96026596 & -0.39468800 & 1.00000000 & -0.00399999 & $3.97 \mathrm{E}-02$ & $3.91 \mathrm{E}-01$ \\
\hline 0.003 & 0.91134193 & -0.58216130 & 1.00000000 & -0.00599997 & $8.87 \mathrm{E}-02$ & $5.76 \mathrm{E}-01$ \\
\hline 0.004 & 0.84422141 & -0.75801080 & 1.00000000 & -0.00799997 & $1.56 \mathrm{E}-01$ & $7.5 \mathrm{E}-01$ \\
\hline 0.005 & 0.76024460 & -0.91872540 & 1.00000000 & -0.00998752 & $2.40 \mathrm{E}-01$ & $9.09 \mathrm{E}-01$ \\
\hline 0.006 & 0.66108821 & -1.06109600 & 1.00000000 & -0.01199998 & $3.39 \mathrm{E}-01$ & $1.05 \mathrm{E} 00$ \\
\hline 0.007 & 0.54873208 & -1.18228010 & 1.00000000 & -0.01399966 & $4.51 \mathrm{E}-01$ & $1.17 \mathrm{E} 00$ \\
\hline 0.008 & 0.42541959 & -1.27985800 & 1.00000000 & -0.01599949 & $5.75 \mathrm{E}-01$ & $1.26 \mathrm{E} 00$ \\
\hline 0.009 & 0.29361288 & -1.35188130 & 1.00000000 & -0.01799927 & $7.06 \mathrm{E}-01$ & $1.33 \mathrm{E} 00$ \\
\hline 0.010 & 0.99001665 & -0.19933400 & 1.00000000 & -0.01999999 & $9.98 \mathrm{E}-03$ & $1.79 \mathrm{E}-01$ \\
\hline 0.020 & 0.96026596 & -0.39468800 & 1.00000000 & -0.03999200 & $3.97 \mathrm{E}-02$ & $3.55 \mathrm{E}-01$ \\
\hline 0.030 & 0.91134193 & -0.58216130 & 1.00000000 & -0.05997302 & $8.87 \mathrm{E}-02$ & $5.22 \mathrm{E}-01$ \\
\hline 0.040 & 0.84422141 & -0.75801080 & 1.00000010 & -0.07993605 & $1.56 \mathrm{E}-01$ & $6.78 \mathrm{E}-01$ \\
\hline 0.050 & 0.76024460 & -0.91872540 & 1.00000020 & -0.09987515 & $2.40 \mathrm{E}-01$ & $8.19 \mathrm{E}-01$ \\
\hline 0.060 & 0.66108821 & -1.06109600 & 1.00000040 & -0.11978440 & $3.39 \mathrm{E}-01$ & $9.41 \mathrm{E}-01$ \\
\hline 0.070 & 0.54873208 & -1.18228010 & 1.00000080 & -0.13965780 & $4.51 \mathrm{E}-01$ & $1.04 \mathrm{E} 00$ \\
\hline 0.080 & 0.42541959 & -1.27985800 & 1.00000140 & -0.15948960 & $5.75 \mathrm{E}-01$ & $1.12 \mathrm{E} 00$ \\
\hline 0.090 & 0.29361288 & -1.35188130 & 1.00000220 & -0.17927390 & $7.06 \mathrm{E}-01$ & $1.17 \mathrm{E} 00$ \\
\hline 0.100 & 0.15594369 & -1.39691200 & 1.00000330 & -0.19900490 & $8.44 \mathrm{E}-01$ & $1.20 \mathrm{E} 00$ \\
\hline 0.200 & 0.96026596 & -0.39468800 & 1.00053700 & -0.39215690 & $4.02 \mathrm{E}-02$ & $2.53 \mathrm{E}-03$ \\
\hline 0.300 & 0.91134193 & -0.58216130 & 1.00274900 & -0.57416270 & $9.14 \mathrm{E}-02$ & $7.80 \mathrm{E}-03$ \\
\hline 0.400 & 0.84422141 & -0.75801080 & 1.00884500 & -0.74074070 & $1.65 \mathrm{E}-01$ & $1.73 \mathrm{E}-02$ \\
\hline 0.500 & 0.76024460 & -0.91872540 & 1.02222200 & -0.88888890 & $2.62 \mathrm{E}-01$ & $2.98 \mathrm{E}-02$ \\
\hline 0.600 & 0.66108821 & -1.06109600 & 1.04817100 & -1.01694900 & $3.87 \mathrm{E}-01$ & $4.41 \mathrm{E}-02$ \\
\hline 0.700 & 0.54873208 & -1.18228010 & 1.09547100 & -1.12449800 & $5.47 \mathrm{E}-01$ & $5.78 \mathrm{E}-02$ \\
\hline 0.800 & 0.42541959 & -1.27985800 & 1.18040900 & -1.21212100 & $7.55 \mathrm{E}-01$ & $6.77 \mathrm{E}-02$ \\
\hline 0.900 & 0.29361288 & -1.35188130 & 1.33838800 & -1.28113900 & $1.04 \mathrm{E} 00$ & $7.07 \mathrm{E}-02$ \\
\hline 1.000 & 0.15594369 & -1.39691200 & 1.66666700 & -1.33333300 & $1.51 \mathrm{E} 00$ & $6.36 \mathrm{E}-02$ \\
\hline
\end{tabular}


International Journal of Engineering Applied Sciences and Technology, 2020

Vol. 4, Issue 9, ISSN No. 2455-2143, Pages 104-115

Published Online January 2020 in IJEAST (http://www.ijeast.com)

Table 2: Solution to Example 2 at $\mathrm{h}=0.001$

\begin{tabular}{|c|c|c|c|c|c|c|}
\hline & Exact & Solutions & \multicolumn{1}{l|}{ Approximated } & \multicolumn{1}{l|}{ Solutions } & \multicolumn{2}{l|}{ Error } \\
\hline $\mathrm{H}$ & $\mathrm{y}(\mathrm{x})$ & $\mathrm{y}^{\prime}(\mathrm{x})$ & $\mathrm{y}(\mathrm{x})$ & $\mathrm{y}^{\prime}(\mathrm{x})$ & $\mathrm{y}$ & $\mathrm{y}^{\prime}$ \\
\hline 0.001 & 1.00000050 & 0.00100000 & 1.00000000 & 0.00100000 & $5.00 \mathrm{E}-07$ & $0.00 \mathrm{E} 00$ \\
\hline 0.002 & 1.00000200 & 0.00200000 & 1.00000100 & 0.00200000 & $1.00 \mathrm{E}-06$ & $0.00 \mathrm{E} 00$ \\
\hline 0.003 & 1.00000450 & 0.00300001 & 1.00000100 & 0.00300000 & $3.50 \mathrm{E}-06$ & $1.00 \mathrm{E}-09$ \\
\hline 0.004 & 1.00000800 & 0.00400003 & 1.00000300 & 0.00400003 & $5.00 \mathrm{E}-06$ & $0.00 \mathrm{E} 00$ \\
\hline 0.005 & 1.00001250 & 0.00500006 & 1.00000400 & 0.00500006 & $8.50 \mathrm{E}-06$ & $0.00 \mathrm{E} 00$ \\
\hline 0.006 & 1.00001800 & 0.00600011 & 1.00000600 & 0.00600011 & $1.20 \mathrm{E}-05$ & $0.00 \mathrm{E} 00$ \\
\hline 0.007 & 1.00002450 & 0.00700017 & 1.00000800 & 0.00700017 & $1.65 \mathrm{E}-05$ & $0.00 \mathrm{E} 00$ \\
\hline 0.008 & 1.00003200 & 0.00800026 & 1.00001100 & 0.00800026 & $2.10 \mathrm{E}-05$ & $0.00 \mathrm{E} 00$ \\
\hline 0.009 & 1.00004050 & 0.00900037 & 1.00001400 & 0.00900037 & $2.65 \mathrm{E}-05$ & $0.00 \mathrm{E} 00$ \\
\hline 0.010 & 1.00000050 & 0.00100000 & 1.00001700 & 0.01000050 & $1.65 \mathrm{E}-05$ & $9.00 \mathrm{E}-03$ \\
\hline 0.020 & 1.00000200 & 0.00200000 & 1.00006700 & 0.02000400 & $6.50 \mathrm{E}-05$ & $1.80 \mathrm{E}-02$ \\
\hline 0.030 & 1.00000450 & 0.00300001 & 1.00015000 & 0.03001351 & $1.46 \mathrm{E}-04$ & $2.70 \mathrm{E}-02$ \\
\hline 0.040 & 1.00000800 & 0.00400003 & 1.00026700 & 0.04003202 & $2.60 \mathrm{E}-04$ & $3.60 \mathrm{E}-02$ \\
\hline 0.050 & 1.00001250 & 0.00500006 & 1.00041800 & 0.05006256 & $4.06 \mathrm{E}-04$ & $4.51 \mathrm{E}-02$ \\
\hline 0.060 & 1.00001800 & 0.00600011 & 1.00060200 & 0.06010815 & $5.84 \mathrm{E}-04$ & $5.41 \mathrm{E}-02$ \\
\hline 0.070 & 1.00002450 & 0.00700017 & 1.00082000 & 0.07017181 & $7.96 \mathrm{E}-04$ & $6.32 \mathrm{E}-02$ \\
\hline 0.080 & 1.00003200 & 0.00800026 & 1.00107200 & 0.08025661 & $1.04 \mathrm{E}-03$ & $7.23 \mathrm{E}-02$ \\
\hline 0.090 & 1.00004050 & 0.00900037 & 1.00135900 & 0.09036561 & $1.32 \mathrm{E}-03$ & $8.14 \mathrm{E}-02$ \\
\hline 0.100 & 1.00005000 & 0.01000050 & 1.00501900 & 0.10050190 & $4.97 \mathrm{E}-03$ & $9.05 \mathrm{E}-02$ \\
\hline
\end{tabular}

